\title{
Modeling of non-Newtonian blood flow through a stenosed artery incorporating fluid-structure interaction
}

\author{
W. Y. Chan* Y. Ding ${ }^{\dagger} \quad$ J. Y. Tu*
}

(Received 21 November 2005; revised 12 December 2006)

\begin{abstract}
We investigate fluid and structural responses to pulsatile nonNewtonian blood flow through a stenosed artery, using AnsYs. The artery was modeled as an axisymmetric stenosed vessel. The wall of the vessel was set to be isotropic and elastic. The blood behavior was described by the Power Law and the Carreau non-Newtonian models, respectively. When compared to the Newtonian flow models, the result from the Carreau model showed very little difference, in terms of velocity, pressure and wall shear stress, whereas the result from the
\end{abstract}

*School of Aerospace, Mechanical and Manufacturing Engineering, RMIT University, Melbourne, Australia.

http://www .rmit. edu . au/browse/?QRY=jiyuan+Tu\&STYPE=PEOPLE

${ }^{\dagger}$ School of Mathematical and Geospatial Sciences, RMIT University, Melbourne, AUstRalia. http://www . rmit. edu . au/browse/?QRY=Yan+Ding\&STYPE=PEOPLE

See http://anziamj.austms.org.au/V47EMAC2005/Chan for this article, (C) Austral. Mathematical Soc. 2007. Published January 4, 2007. ISSN 1446-8735 
Power Law model showed more significant vortices and smaller wall shear stresses. The highest stress concentration was also found at the throat of the stenosis.

\section{Contents}

1 Introduction

C508

2 Methods

C510

2.1 Model description . . . . . . . . . . . . . . . . C510

2.2 Governing equations . . . . . . . . . . . . . . C511

2.3 Flow conditions and model settings . . . . . . . . . C512

2.4 Non-Newtonian models . . . . . . . . . . . . . . . . C513

2.5 Computational details . . . . . . . . . . . . . . . C513

\section{Results}

C514

3.1 Validation of rigid and FSI Newtonian simulations . . . . C C515

3.2 FSI results . . . . . . . . . . . . . . . . C C515

3.2.1 Axial velocity profile . . . . . . . . . . C515

3.2 .2 Wall shear stress . . . . . . . . . . . C C516

3.2 .3 Stress and displacement . . . . . . . . . . . C C517

3.2.4 Non-Newtonian importance factor . . . . . . . . C C517

4 Conclusion

C520

References

C521

\section{Introduction}

A stenosed artery is the result of atherosclerosis, that is, the hardening of the artery due to the growth of a calcified plaque layer on the inner walls of the 
artery. Numerous health complications caused by the disease have drawn a significant research interest in the understanding of the cause of the disease in order to develop effective methods to treat the disease.

The earliest research concentrated on the effects of stenosis shape and severity [10] and the effects of transient conditions on the flow [3]. In these early studies, the blood was assumed to be a Newtonian fluid, which is valid for fluids with shear rates greater than $100 \mathrm{~s}^{-1}$. This situation only occurs for a blood flow in large arteries [7]. Since blood flow in small arteries often exhibits non-Newtonian characteristics, how these affect flow behavior has attracted a considerable research interest. In order to determine the most suitable model for simulating the changes of viscosity in blood steam, Cho and Kensey [2] investigated several non-Newtonian models, including Power Law and Carreau models, and compared their results with actual blood samples. Perktold et al. [8] found that the Power Law model demonstrated more significant non-Newtonian influence. Most recently, Johnson and coworkers [4] found that the Carreau model seemed to be far more suitable for blood flow as the results from Carreau model agreed best with most of their experimental data. They also found that the Carreau model did not overpredict the fluid behaviour near the vessel wall for cases of high velocities with a significant non-Newtonian impact on the flow.

Another recent development in this research field is the numerical simulation of fluid-structure interaction (FSI) between a blood flow and diseased arteries. This is of prime importance in predicting where arterial lesions are most likely to occur, because they lead to health complications, such as heart attack and stroke. Another health risk is that the stenosed arteries may collapse due to a low pressure around the stenotic portion [11]. To understand the occurrence of these phenomena, several studies were conducted to model the stress and displacement distributions along the diseased artery wall. Lee and $\mathrm{Xu}$ [5] modeled a Newtonian blood flow past an axisymmetric compliant stenosis with sharp transitions and studied the impact of the flow on the wall of the stenosed tube. The geometry of their model was similar to that 
used in the experiment work of Ojha et al. [6]. Tang et al. [11] also studied the impact of a Newtonian flow on three-dimensional stenotic tubes with different degrees of severity for both axisymmetric and asymmetric conditions. These studie made great contributions to the understanding of the complex phenomena resulted by FSI.

However, there has been a lack of research, in the area of FSI modeling of blood flows in small arteries, in which the non-Newtonian fluid effect can not be ignored. Thus, the objective of this study is to numerically model the interactions between a non-Newtonian fluid and a solid wall with a small elastic deformation. This paper reports the results of fluid and structural responses to a pulsatile non-Newtonian blood flow through an axisymmetric stenosed vessel. Both Carreau and Power Law models were used to study the effects of the non-Newtonian fluid on the artery wall and the elastic deformation of the artery wall on the fluid. The wall stress distributions, fluid velocity profile and wall shear stress were also quantified and are discussed in detail.

\section{Methods}

\subsection{Model description}

This problem concerns an unsteady flow through a constricted (45\% area reduction) elastic tube similar to the case used by Lee and Xu [5]. Initially, two cases were run to validate the geometry and FSI model - a Newtonian pulsatile flow through a rigid wall and a Newtonian pulsatile flow through an elastic wall. Then, these two cases were used to investigate a pulsatile flow through elastic wall using the Carreau and Power Law non-Newtonian models. The geometry of these models is shown in Figure 1, which consists of a cylindrical tube with internal and external diameters of $5 \mathrm{~mm}$ and $6 \mathrm{~mm}$ respectively. 


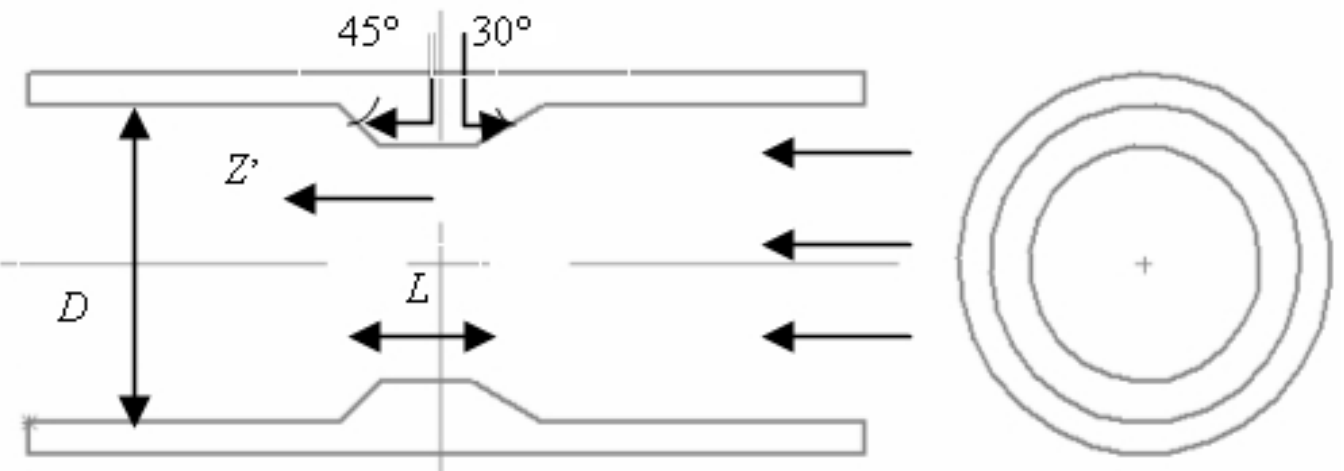

Figure 1: The geometry of the $45 \%$ axisymmetric stenosis used in all the Newtonian and non-Newtonian simulations where $L=1.5 \mathrm{~mm}$ and $D=$ $5.0 \mathrm{~mm}$. The normalised distance from the centre of stenosis is $Z=Z^{\prime} / D$. On the right is a cross-sectional view of the model from the end.

\subsection{Governing equations}

Adopting indicial notation (with the coordinate axes referred as $x_{i}, i=$ $1,2,3)$, the velocity of an fluid flow is denoted as $u_{i}, i=1,2,3$. According to the conservations of mass and momentum, the governing equations for an isotropic, incompressible fluid flow are the continuity equation and momentum equations (ignoring the body forces):

$$
\begin{aligned}
& \frac{\partial u_{i}}{\partial x_{i}}=0 \\
& \frac{\partial u_{i}}{\partial t}+\frac{\partial\left(u_{i} u_{j}\right)}{\partial x_{j}}=\frac{1}{\rho}\left[-\frac{\partial p}{\partial x_{i}}+\frac{\partial \tau_{i j}}{\partial x_{j}}\right],
\end{aligned}
$$

where $p$ is the pressure, $\rho$ is the density of the fluid, and the deviatoric stresses that link to the deviatoric strain rates of the fluid are

$$
\tau_{i j}=2 \mu\left(\dot{\epsilon}_{i j}-\frac{1}{3} \delta_{i j} \dot{\epsilon}_{k k}\right),
$$


where the quantity in the brackets is the deviatoric strain rate, $\delta_{i j}$ is the Kronecker delta, a repeated index implies summation over the range of index, and

$$
\dot{\epsilon}_{i j}=\frac{1}{2}\left[\frac{\partial u_{i}}{\partial x_{j}}+\frac{\partial u_{j}}{\partial x_{i}}\right] .
$$

For a non-Newtonian fluid flow, the non-linear relation between the stress and strain rate is observed with a coefficient $\mu$ depending on strain rates as discussed in Section 2.4.

The motion of an elastic solid is governed by

$$
\rho_{w} \frac{\partial^{2} d_{i}}{\partial t^{2}}=\frac{\partial \sigma_{i j}}{\partial x_{j}},
$$

where $d_{i}$ and $\sigma_{i j}$ are the displacements and stresses of the solid, respectively; and $\rho_{w}$ is the density of the solid wall. The stress tensor $\sigma_{i j}$ is obtained from the constitutive equation of the material, and for a Hookean elastic solid is

$$
\sigma_{i j}=\lambda_{L} e_{k k} \delta_{i j}+2 \mu_{L} e_{i j},
$$

where $\lambda_{L}$ and $\mu_{L}$ are the Lame constants, and $e_{i j}$ are the strain in the solid.

These equations were solved using FLOTRAN and ANSYS codes available within ANSYS/Multiphysics.

\subsection{Flow conditions and model settings}

For the preliminary Newtonian models, pulsatile flow was specified at the inlet as a sinusoidal volumetric flow waveform of $4.3 \pm 2.6 \mathrm{ml}$ with a period $t_{p}=345 \mathrm{~ms}$. The fluid has a density of $755 \mathrm{~kg} \mathrm{~m}^{-3}$ and a Newtonian viscosity of $0.00143 \mathrm{~N} \mathrm{~s} \mathrm{~m}^{-2}$. giving the maximum and minimum Reynolds number Re to be 930 and 230, respectively. Because of the low Re and mild stenosis the flow is laminar and we assume axisymmetry. For these models 
a fully developed axial velocity profile calculated from the time dependent flow rate was specified at the inlet and a constant pressure of $4140 \mathrm{~Pa}$ was specified for the outlet boundary.

For the FSI model, the stenotic wall was considered to be incompressible, isotropic and linearly elastic, with a Young's modulus of $500 \mathrm{kPa}$, a Poisson ratio of 0.499 , and a density of $1000 \mathrm{~kg} / \mathrm{m}^{3}$.

\subsection{Non-Newtonian models}

Two non-Newtonian models were used to obtain the fluid viscosity, $\mu$, from the strain, $\dot{\gamma}$, which is the second invariant of the deviatoric strain rate tensor of Equation (4). In these models, the fluid density was set as $1050 \mathrm{~kg} / \mathrm{m}^{3}$, the inlet velocity profile was set to be uniform, given in Poise $P(1 P=$ $0.1 \mathrm{Ns} / \mathrm{m}^{2}$ ) [4]. The two non-Newtonian models are the

$$
\text { Carreau model, } \quad \mu=\mu_{\infty}+\left(\mu_{0}-\mu_{\infty}\right)\left[1+(\lambda \dot{\gamma})^{2}\right]^{(n-1) / 2},
$$

where time is constant, $\lambda=3.313 \mathrm{~s}$, zero strain viscosity $\mu_{0}=0.56 \mathrm{P}$, infinite strain viscosity $\mu_{\infty}=0.0345 \mathrm{P}$, empirical exponent $n=0.3568$; and the

$$
\text { Power Law model, } \quad \mu=\mu_{0}(\dot{\gamma})^{n-1} \text {, }
$$

where nominal viscosity $\mu_{0}=0.35$ and empirical exponent $n=0.6$.

For comparison, a FSI Newtonian model was run with the same density and inlet conditions. The viscosity was set to be $0.0345 \mathrm{P}$ (infinite strain viscosity).

\subsection{Computational details}

The $45 \%$ area reduction in the stenosed tube was modeled to be axisymmetric and the flow is likewise assumed to behave as such. As in the case of 
Lee and $\mathrm{Xu}$ [5], the pre- and post-stenotic regions were chosen to be 3 and 10 diameters (that is, $15 \mathrm{~mm}$ and $50 \mathrm{~mm}$ ) long respectively. Several grid densities were tested to determine the optimum computational settings, all using quadrilateral structured elements. There was no notable change between these different refinements. A $25 \times 325$ mesh density was used for the fluid with 12 elements for the solid radial thickness, as shown in Figure 3.

For this study, 69 time steps per cycle were chosen. The SIMPLEF velocitypressure coupling was specified for the FLOTRAN solver. The convergence criteria for all the fluid variables were $10^{-5}$. For the FSI coupling, the convergence criterion for the maximum difference in wall displacement was set to a tolerance of $0.1 \%$ of the diameter.

The FSI coupling method employed in this study consists of an iterative scheme, whereby the fluid model is first solved and the fluid pressures are applied on the inner wall and deformed while solving the solid model, which is then used to update the coordinates of the corresponding nodes. This is repeated until the convergence is achieved on nodal displacements.

\section{$3 \quad$ Results}

The results for the fluid and solid properties, for example, velocity distributions, wall shear stress, pressure, wall stress and displacements, are presented at selected time frames. The parameter $t / t_{p}$ used to describe a particular time in a cycle is similar to that used by Lee and $\mathrm{Xu}$, where $t$ is the time in seconds and the period of the cycle $t_{p}=0.345 \mathrm{~s}$.

Lee and $\mathrm{Xu}[5]$ compared their flow model with experimental data obtained by Ojha et al. [6] at three different locations in the post-stenotic region: $Z^{\prime}=1, Z^{\prime}=2.5$ and $Z^{\prime}=4.3$, where $Z^{\prime}$ is the normalised distance from the centre of the stenosis; that is, $Z^{\prime}=z / D$, and $z$ is the axial distance away from the centre of the stenosis and $D$ is the inlet diameter of the tube 
(Figure 1). The flow waveform specified at the model inlet is similar to Lee and Xu's case [5], which has a time-shift of $0.123 \mathrm{~s}$ with the experiment by Ojha et al. [6].

\subsection{Validation of rigid and FSI Newtonian simulations}

To validate the model and methods employed in this study, a comparison with the studies by Lee and Xu [5] and Ballyk et al. [1] was conducted [9]. Good agreement with the numerical and experimental studies was found. We concluded that incorporating FSI into the simulation increased the flow crosssectional area in the non-stenosed regions, resulting in reducing the velocity profile and increasing the flow recirculation effects: the wall shear stress was decreased in the FSI model compared to the rigid model; the maximum stress was found at the shoulder of the stenosis.

\subsection{FSI results}

\subsubsection{Axial velocity profile}

Figure 2 shows that the Carreau model velocity profiles mostly follow the profiles of the Newtonian except for flow at a slightly slower velocity. Close to the centreline, the velocity of the Carreau model noticeably drops further, and this effect is more apparent further downstream from the stenosis. We attribute this fluid behavior to the higher viscosity of the fluid, particularly close to the centreline region where the shear rate is lowest.

The velocity profiles for the Power Law model show a marked decrease in centreline axial velocities in comparison to the Newtonian model. Due to a steeper change in the viscosity near the artery wall, the boundary layer 


$$
Z^{\prime}=1
$$

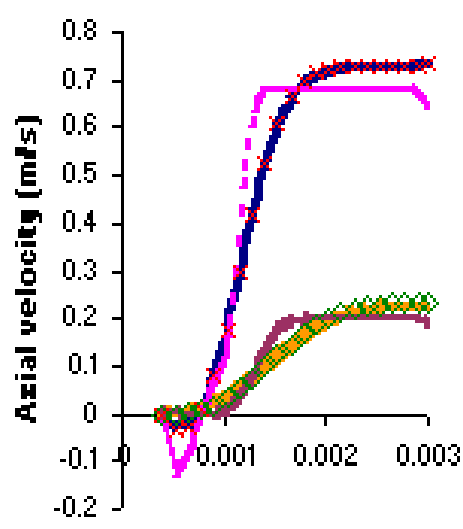

Radial distance $(\mathbf{m})$
$Z^{\prime}=\mathbf{4 . 3}$

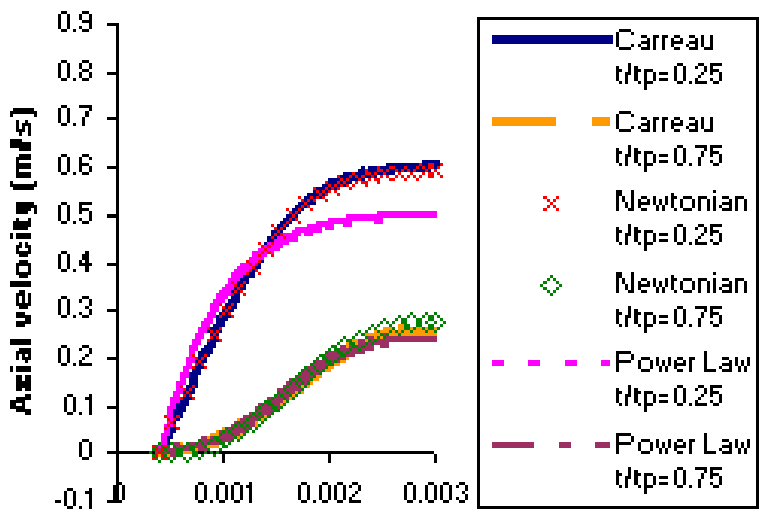

Radial distance $(\mathbf{m})$

Figure 2: Comparison of axial velocity profiles between the Carreau, Power Law and Newtonian FSI models at $Z^{\prime}=1$ and $Z^{\prime}=4.3$.

thickness is much thinner than for the other models, which causes a flatter profile and a lower centreline axial velocity. The largest difference in centreline axial velocities occurs at the peak of the acceleration phase which gradually decreases during the deceleration phase reaching the minimum. The recirculation zone was slightly less prominent for the Power Law model.

\subsubsection{Wall shear stress}

The wall shear stress (wss) for the Carreau model (Figure 3) shows the four transitional points and characteristic peaks and troughs noted by Lee and $\mathrm{Xu}[5]$. The Carreau model indicated slightly higher wss distributions than the Newtonian model due to the higher viscosity near the walls. This observation is consistent with results of Johnston et al. [4] who noted this wss behavior for the Carreau model. 
The wall shear stress for the Power Law model has similar peaks and troughs as shown in the Carreau model. However, the overall wss is significantly lower than the other models. This result is supported by Johnston et al. [4] who noted that at high centreline velocities, the Power Law model tends to have much lower wss than other models.

\subsubsection{Stress and displacement}

The stress distribution (Figure 4) for this model remains similar throughout the flow cycle as the pressure has little variation over the cycle and the dominance of the singularity points. Therefore contours at only one time are presented. From the stress contours, the highest stress is the axial stress and occurs on the shoulders of the stenosis, particularly downstream where the bending effect is significant. The relatively sharp corner of the bend contributes to a stress concentration due to solution singularity. Circumferential stress magnitudes are close to the value for the axial stresses but are due to the constraints imposed by the axisymmetric condition. Radial stresses are not as significant and are less likely to cause surface rupture.

Due to the similar magnitudes in pressure, the stress distributions for the Power Law model have the same distribution as the Carreau model over time, having the highest stress at the shoulder of the stenosis. However, it is noted that the Power Law stress distributions have a slightly smaller magnitude than the Carreau and Newtonian values.

\subsubsection{Non-Newtonian importance factor}

One method to determine the effect of the non-Newtonian model, proposed by Johnston et al. [4], is the importance factor $I_{L}$ which is derived from the concept introduced by Ballyk et al. [1] who defined this as $I_{L}=\bar{\mu}_{\text {eff }} / \mu_{\infty}$ where $\bar{\mu}_{\text {eff }}$ is the effective viscosity characteristic of a particular flow and 


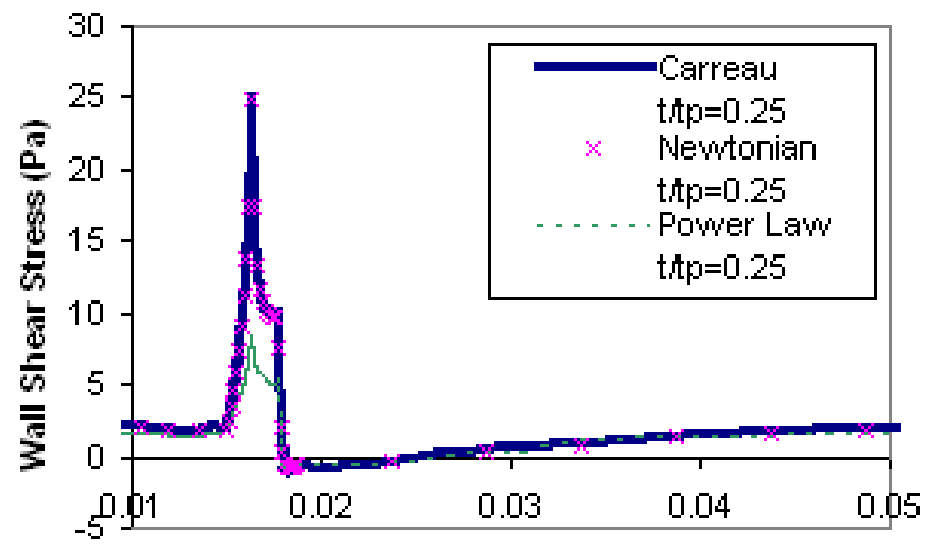

Axial Distance (m)

Figure 3: wsS distribution along the axial distance for the Carreau, Power Law and Newtonian FSI models at $t / t_{p}=0.25$

(a)

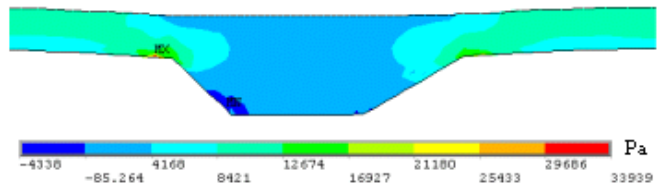

(c)

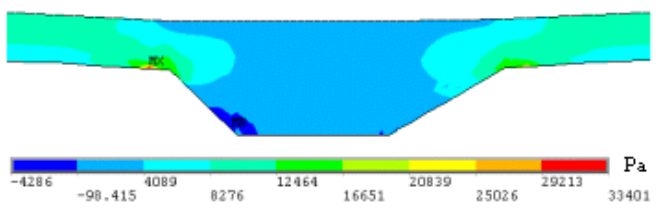

(b)

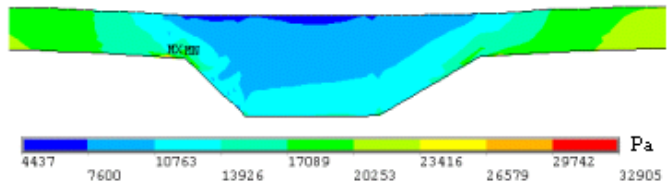

(d)

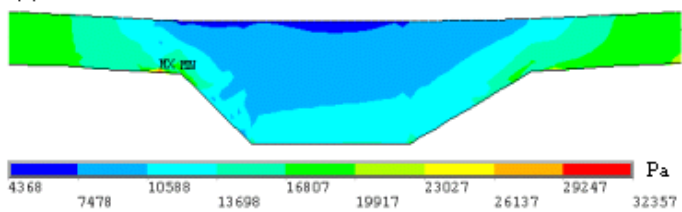

Figure 4: Stress contour distributions of the vessel wall at the stenosed region for the Carreau FSI model in the (a) axial, and (b) circumferential directions and the Power Law FSI model in the (c) axial, and (d) circumferential directions at $t / t_{p}=0.25$. 
TABLE 1: Importance factors for both the Careau and Power Law FSI models. The two areas of interest used to derive these values are the entire flow region and the region immediately on the vessel wall.

\begin{tabular}{|cc|cc|cc|}
\hline & & \multicolumn{2}{|c|}{ Careau } & \multicolumn{2}{c|}{ Power Law } \\
$\frac{t}{t_{p}}$ & $V\left[\mathrm{~m} \mathrm{~s}^{-1}\right]$ & Whole model & Wall only & Whole model & Wall only \\
\hline 0.25 & 0.345 & 1.74652 & 0.80036 & 2.71559 & 2.94888 \\
0.5 & 0.219 & 1.45356 & 2.16144 & 2.30512 & 11.5215 \\
0.75 & 0.087 & 1.22014 & 2.34278 & 2.18004 & 9.62648 \\
1.0 & 0.219 & 1.28388 & 0.63324 & 2.26387 & 2.78711 \\
\hline
\end{tabular}

$\mu_{\infty}=0.00345 \mathrm{~N} \mathrm{~m} \mathrm{~s}^{-1}$ is the Newtonian value for viscosity. Clearly, $I_{L}=1$ indicates Newtonian flow and deviations from unity indicate regions of nonNewtonian flow. Johnston et al. improved on this concept by calculating an average of these importance values that would be more representative of the actual flow in the artery. Instead of simply averaging $I_{L}$, they averaged the relative difference of each value of viscosity from the Newtonian value that is then expressed as a percentage. This global non-Newtonian importance factor

$$
I_{G}=\frac{100}{N} \frac{\left[\sum_{N}\left(\mu-\mu_{\infty}\right)^{2}\right]^{1 / 2}}{\mu_{\infty}} .
$$

This equation is evaluated at each of the $\mathrm{N}$ nodes on the area of interest with $\mu$ as the viscosity, $\mu_{\infty}=0.00345 \mathrm{~N} \mathrm{~m} \mathrm{~s}^{-1}$. For the non-Newtonian simulations, the global importance factor is tabulated in Table 1.

Considering the whole model, both the Carreau model and the Power Law model indicate an extremely high $I_{G}$ at $t / t_{p}=0.25$, the peak of the fluid flow. This value decreases as the flow decelerates to the point of its minimum, after which the $I_{G}$ picks up again when the flow accelerates. Between the Power Law and Carreau model, it seems that the Power Law model has larger deviations from the Newtonian viscosity due to the nature of the equation that has a steeper strain-viscosity relation. This occurrence has been noted by Johnston et al. [4]. Considering the whole model, both the Carreau model 
and the Power Law model indicate an extremely high $I_{G}$ at $t / t_{p}=0.25$, the peak of the fluid flow. This value is seen to decrease as the flow decelerates to the point of minimum flow, after which, the $I_{G}$ picks up again when the flow accelerates. Between the Power Law and Carreau model, it would seem that the Power Law model has larger deviations from the Newtonian viscosity due to the nature of the equation that has a steeper strain-viscosity relation. This occurrence has been noted by Johnston et al. [4]. It appears that the Carreau model provides reasonable $I_{G}$ values without being too excessive. Note: despite having similar velocities at $t / t_{p}=0.5$ and $t / t_{p}=1.0$, the $I_{G}$ differs at these times. This indicates that the temporal nature of the flow can affect the impact the non-Newtonian model has on the flow and that this impact is larger during flow deceleration where the recirculation zone is seen to grow. It appears that the Carreau model provides reasonable $I_{G}$ values without being too excessive. Also note that despite having similar velocities at $t / t_{p}=0.5$ and $t / t_{p}=1.0$, the $I_{G}$ differs at these times. This indicates that the temporal nature of the flow can affect the impact the non-Newtonian model has on the flow and that this impact is larger during flow deceleration where the recirculation zone is seen to grow.

When comparing the wall-only $I_{G}$ to the entire model, the wall-only values were significantly higher and independent of the flow. The wall-only values during the deceleration phase are three times higher than the values during the acceleration phase due to the significant viscosity changes about this region.

\section{Conclusion}

From the results of the Newtonian FSI and rigid simulation, the model used is sufficiently accurate. These results show that having a compliant model has a slight effect on the flow properties due to the enlargement of the flow cross-sectional area that causes a reduction in the flow. The models showed 
different WSS peak magnitudes, but the overall shape of the distribution remained similar.

The Carreau model showed only slightly smaller centreline axial velocities whereas the Power Law model showed more significant differences, including flatter velocity profiles. This was due to the higher viscosity about the axis of symmetry, particularly for the Power Law model. The wss distributions show similar trends except the Power Law model shows significantly smaller magnitudes. Both models show similar stress distributions although the Carreau exhibits slightly larger stresses.

The values of importance factors indicate the impact of the non-Newtonian fluid in various flow regions. Transient effects are apparent in the values. There is a marked decrease in importance factors during the deceleration phase. The wall-only importance factors indicate a significant nonNewtonian effect.

Though the Power Law model shows more dominant non-Newtonian effects, it may produce excessive values, according to literature. Further experimental work is recommended to determine which model is more suitable for this type of simulation.

\section{References}

[1] P. D. Ballyk, D. A. Steinman and C. R. Ethier. Simulation of non-Newtonian blood flow in an end-to-side anastomosis. Biorheology, 44(5):565-586, 1994. C515, C517

[2] Y. I. Cho and K. R. Kensey. Effects of the non-Newtonian viscosity of blood on hemodynamics of diseased arterial flows. Advances in Bioengineering, BED 15:147-148, 1989. C509 
[3] K. Imaeda and F. O. Goodman. Analysis of nonlinear pulsatile blood flow in arteries. Journal of Biomechanics, 13:165-174: 1980. C509

[4] B. M. Johnston, P. R. Johnston, S. Corney and D. Kilpatrick. Non-Newtonian blood flow in human right coronary arteries: steady state simulations. Journal of Biomechanics, 37: 709-720, 2004. doi:10.1016/j.jbiomech.2003.09.016 C509, C513, C516, C517, C519, C520

[5] K. W. Lee and X. Y. Xu. Modelling of flow and wall behaviour in a mildly stenosed tube. Medical Engineering \&6 Physics, 24:575-586, 2002. doi:10.1016/S1350-4533(02)00048-6 C509, C510, C514, C515, C516

[6] M. Ojha, R. S. C. Cobbold, K. W. Johnston and R. L. Hummel. Pulsatile flow through constricted tubes: an experimental investigation using photochromic tracer methods. Journal of Fluid Mechanics, 203:173-197, 1989. C510, C514, C515

[7] T. J. Pedley. The Fluid Mechanics of Large Blood Vessels, Cambridge University Press, 1980. C509

[8] K. Perktold, R. Peter and M. Resch. Pulsatile non-Newtonian blood flow simulation through a bifurcation with an aneurysm. Biorheology, 26:1011-1030, 1989. C509

[9] W. Y. Chan, Simulation of Arterial Stenosis Incorporating Fluid Structural Interaction and non Newtonian Blood Flow. Master's Thesis, RMIT University, Australia, 2006. C515

[10] F. T. Smith. The separation flow through a severely constricted symmetric tube. Journal of Fluid Mechanics, 90: 725-754, 1979. C509

[11] D. Tang, C. Yang, S. Kobayashi and D. N. Ku. Generalized finite difference method for 3-D viscous flow in stenotic tubes with large wall 
deformation and collapse. Applied Numerical Mathematics, 38: 49-68, 2001. doi:10.1016/S0168-9274(00)00062-3 C509, C510 